\title{
How Frequent is Evolutionary Spin-Up in Binary Be Stars?
}

\author{
M. Maintz ${ }^{1}$, Th. Rivinius ${ }^{1,2}$, S. Stefl ${ }^{3}$, and O. Stahl ${ }^{1}$ \\ ${ }^{1}$ Landessternwarte Königstuhl, D-69117 Heidelberg, Germany \\ ${ }^{2}$ ESO, Karl-Schwarzschild-Str. 2, D-85748 Garching, Germany \\ ${ }^{3}$ Astronomical Institute, Academy of Sciences, CZ-25165 Ondřejov, \\ Czech Republic
}

\begin{abstract}
Satellite absorptions are identified as a general feature of Be binaries. In addition, $\mathrm{Be}+\mathrm{sdO}$ binaries seem to show a characteristic short-term emission variability and short-lived shell events. The identification of such features can ease the search for further Be binaries with hot companions to allow an estimate of the fraction of Be stars, possibly spun-up through binary evolution.
\end{abstract}

\section{Introduction}

Be stars are among the most rapid rotators, with about $70 \%$ of the critical rate. Assuming standard binary evolution scenarios, a non-negligible fraction of Be stars might have underwent spin-up through mass transfer, leading either to $\mathrm{Be}+\mathrm{WD}$ or $\mathrm{Be}+\mathrm{sdO}$ systems. Yet, only a single confirmed $\mathrm{Be}+\mathrm{sdO}$ pair, $\phi$ Per, is known, although Be stars belong to the most intensively studied objects.

\section{Features Indicating $\mathrm{Be}+\mathrm{sdO}$ Binaries}

Many Be stars, archived in our data base, show specific features occuring as small absorption cores in the peaks of emission components of e.g. Balmer lines at special orbital phases (Fig. 1, left). Almost all (except one) of these stars, like $\phi$ Per, HR 2142, and $\kappa$ Dra, are confirmed Be binaries. Therefore these features are taken as indicators for binarity.

$\phi$ Per, 59 Cyg, and HR 2142 in addition show a specific short-term variability of emission lines, varying periodically in anti-phase to the photospheric absorption (Fig. 1, mid). It was first observed in $\phi$ Per (Poeckert 1981) and successfully modeled for He I 5876 and He I 6678 by Hummel \& Štefl (2001), assuming a hot companion, heating and ionizing a sector of the Be disk. It is therefore attributed to a $\mathrm{Be}+\mathrm{sdO}$ nature. The heated area causes extra emission, appearing as a blue- or red shifted emission peak. Its velocity variation represents the orbital velocity of the outer limb of the disk, excited at different phase angles. Gies et al. (1998) found good indications for a sdO companion in the UV spectrum of $\phi$ Per.

A binary nature is also confirmed for $59 \mathrm{Cyg}$. The variation of its He I 6678 emission was successfully reproduced by the hot companion model of Hummel, too, being a strong hint to a further $\mathrm{Be}+$ sdO binary. 

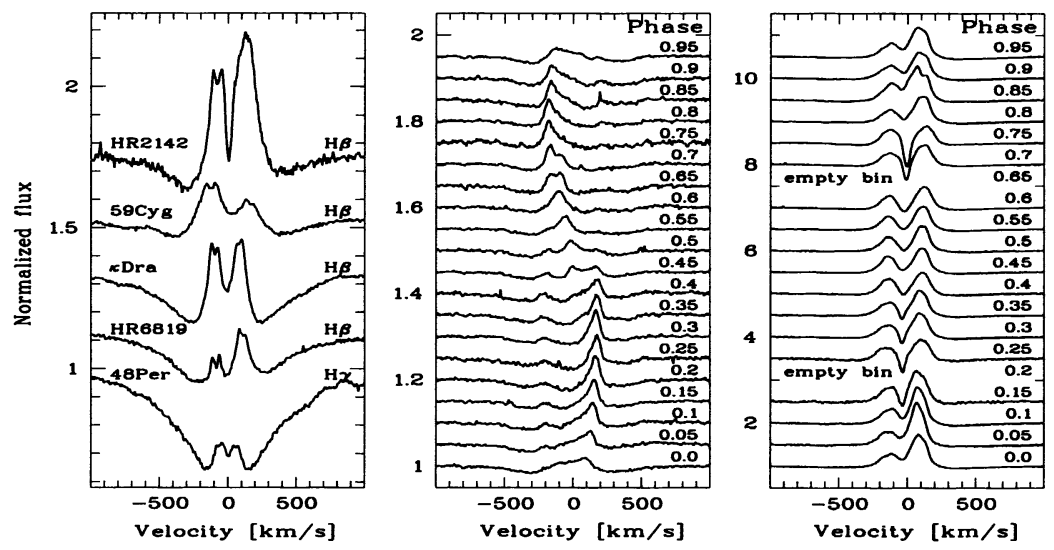

Figure 1. Satellite absorptions found in the spectra of confirmed and supposed Be binaries (left). Characteristic features of $\mathrm{Be}+\mathrm{sdO}$ binaries: emission variability of He 6678 in $59 \mathrm{Cyg}$ (mid), shell event in $\mathrm{H} \beta$ in $\phi$ Per (right).

Phase 0.0 refers to superior conjunction of the primary.

$\phi$ Per and HR 2142, supposed to be seen nearly edge-on, exhibit short-lived shell events occuring periodically around superior conjunction of the primary (Fig. 1, right). They are explicable by the heated sector seen in front of the primary. The companion of the well studied binary HR 2142 (Peters 1983) could not be identified yet. Since it shows the same characteristic features like $\phi$ Per and $59 \mathrm{Cyg}$, it is a good candidate for a third $\mathrm{Be}+\mathrm{sdO}$ binary.

\section{Conclusion}

Satellite absorptions occur in the spectra of many Be stars archived in our data base. Since almost all of them are confirmed binaries, these features are taken as indicators for binarity. Short-term emission variability and short-lived shell events seem to be characteristic for $\mathrm{Be}+\mathrm{sdO}$ binaries. They occur in the spectra of the confirmed $\mathrm{Be}+\mathrm{sdO}$ binary $\phi$ Per and HR 2142, for which such a nature is not verified yet. Since 59 Cyg has a lower inclination, only short-term emission variability is seen. This could also be successfully reproduced by the hot companion model of Hummel, like for $\phi$ Per, indicating 59 Cyg to be another $\mathrm{Be}+$ sdO binary. The identification of such features shall result in selection criteria for further $\mathrm{Be}+\mathrm{sdO}$ systems to concentrate time-consuming monitoring campaigns on promising candidates only. This should permit reliable future estimates of the fraction of Be stars, possibly having spun-up through binary evolution.

\section{References}

Gies, D.R., Bagnuolo, W.G., Jr., Ferrara, E.C. et al. 1998, ApJ 493, 440

Hummel, W., Štefl, S. 2001, A\&A 368, 471

Peters, G. 1983, PASP 95, 311

Poeckert, R. 1981, PASP 93, 297 